\title{
FREQUENCY GUIDED PHASE UNWRAPPING FOR IMPROVED AM-FM RECONSTRUCTION
}

\author{
Patrick A. Campbell and Joseph P. Havlicek
}

\author{
School of Electrical and Computer Engineering, University of Oklahoma, Norman, OK, USA
}

\begin{abstract}
We introduce a new phase unwrapping algorithm that makes it possible to obtain high fidelity image reconstructions from computed AM-FM models without the need for storing multiple boundary conditions such as phase samples from the original image in order to reconstruct the phase from the estimated FM field. This is important to the development of general modulation domain filters because the phase initial conditions are unknown after a filtering operation that modifies the FM functions, making reconstruction of the filtered image hard. In the new approach, frequency information from an initial least squares estimate of the unwrapped phase is used to guide selection of refined phase values that are congruent with the principal phase of the image. The selection process applies a queue-based region growing strategy to compute the final unwrapped phase solution with sparse branch cuts that tend to be placed only in areas with low visual impact. This final solution for the unwrapped phase leads to new solutions for the frequency modulations of the image that are in good agreement with visual perception and provide high quality reconstruction of the original image.
\end{abstract}

Index Terms - AM-FM image models, phase unwrapping, modulation domain signal processing, multicomponent models

\section{INTRODUCTION}

AM-FM models provide a robust, dense characterization of the local nonstationary structure of an image. They have been used with success in important applications including segmentation [1], content based image retrieval [2], target tracking [3,4], and medical image analysis $[5,6]$. An image $t$ is represented as a sum of AM-FM functions according to [7]

$$
t(\mathbf{x})=\sum_{k=1}^{K} t_{k}(\mathbf{x})=\sum_{k=1}^{K} a_{k}(\mathbf{x}) \cos \left[\varphi_{k}(\mathbf{x})\right]
$$

where $a_{k}(\mathbf{x})$ are the amplitude modulation (AM) functions (describing local contrast) and $\nabla \varphi_{k}(\mathbf{x})$ are the frequency modulation (FM) functions (describing local surface pattern granularity and orientation). For a discrete image $t(\mathbf{n})$, agreement with (1) may be obtained by fitting the pixel values with analytically differentiable continuous interpolating functions such as splines [8] to construct an invertible (i.e., perfect reconstruction, or $P R$ ) AM-FM transform. This has enabled the development of modulation domain filters, in which a filtered image is reconstructed from modified AM and FM functions to achieve signal processing goals such as frequency selective attenuation [9], rotation, and scaling [10,11] which would be difficult or impossible to achieve by traditional filtering methods.

This work was supported in part by the U.S. Army Research Laboratory and the U.S. Army Research Office under grant W911NF-08-1-0293.
Naïve computation of $\varphi_{k}(\mathbf{x})$ in (1) by inverse trigonometric functions (arccos for a real image or arctan for a complex image $-c f$. Section 2) results in a wrapped phase that is mathematically correct in the sense that it provides PR, but contains sharp discontinuities along branch cuts of the multi-valued inverse trigonometric function. These phase discontinuities are undesirable because they arise solely from mathematics and are both unrelated to and inconsistent with visual perception of the image. In order to obtain a representation that provides a visually meaningful interpretation of the FM functions as phase gradient, $\varphi_{k}(\mathbf{x})$ must be unwrapped to remove these branch cuts to the extent possible. However, in order to maintain PR, the unwrapped phase at every pixel must be be congruent with the principal phase values in (1).

Prior AM-FM image reconstruction techniques have required saving samples of the phase from the original image to be used as initial conditions for reconstructing $\varphi_{k}$ from the FM gradient field $\nabla \varphi_{k}$ [7-11]. This can lead to serious problems when it is desired for a modulation domain filter to modify the FM functions of the image - because the phase of the filtered image is unknown after FM processing and the phase initial conditions must therefore be estimated or inferred somehow in order to reconstruct the filtered image.

In this paper, we introduce a new phase unwrapping method based on region growing that provides high quality image reconstruction from only a single saved phase sample. In cases where FM filtering has been applied, this simplifies the reconstruction problem since the phase must be estimated at only a single pixel instead of at multiple pixels. Alternatively, if multiple phase samples are saved, then the new phase unwrapping method provides an improved reconstruction quality relative to existing AM-FM reconstruction techniques.

\section{BACKGROUND}

While the AM functions $a_{k}(\mathbf{x})$ and FM functions $\nabla \varphi_{k}(\mathbf{x})$ in (1) are ambiguous for any real-valued image $t(\mathbf{x})$, they are unique for a complex-valued image $z(\mathbf{x})$ once the decomposition into components has been specified [7]. Here, we consider the complex-valued analytic image $z(\mathbf{x})$ obtained by applying the partial Hilbert transform according to $z(\mathbf{x})=t(\mathbf{x})+j \mathcal{H}[t(\mathbf{x})]$ [12]. Decomposition of the complex image $z(\mathbf{x})$ into components is accomplished using an adapted steerable pyramid [13] as described in [12], typically with $K=40$ components $z_{k}(\mathbf{x})=a_{k}(\mathbf{x}) \exp \left[j \varphi_{k}(\mathbf{x})\right]$. The amplitude and phase functions in (1) may then be obtained via

$$
a_{k}(\mathbf{x})=\left|z_{k}(\mathbf{x})\right|
$$

and

$$
\varphi_{k}(\mathbf{x})=\arctan \left(\frac{\operatorname{Im}\left[z_{k}(\mathbf{x})\right]}{\operatorname{Re}\left[z_{k}(\mathbf{x})\right]}\right),
$$

where (3) gives the wrapped phase of each component, also referred to as the principal phase. Once each $\varphi_{k}$ is unwrapped, the 
unwrapped phase $\widehat{\varphi}_{k}$ may be analytically differentiated to obtain a visually meaningful FM function $\nabla \widehat{\varphi}_{k}$. In tandem, the AM and FM functions of all components richly describe the visual image content.

The problem of phase unwrapping has been extensively studied. It is encountered in, e.g., synthetic aperture radar (SAR) [14, 15], magnetic resonance imaging [16], acoustic imaging, projection and diffraction tomography, and adaptive optics [17]. In many applications, the objective is to derive useful information from analysis of the unwrapped phase. However, there is an additional constraint for phase information that is unwrapped within the context of the AMFM image model in order to allow for PR of the original image: each value $\widehat{\varphi}_{k}(\mathbf{x})$ of the unwrapped phase must be congruent with the principal phase $\varphi_{k}(\mathbf{x})$ up to an integer multiple of $2 \pi$ :

$$
\widehat{\varphi}_{k}(\mathbf{x})=\varphi_{k}(\mathbf{x})+2 \pi b, b \in \mathbb{Z} \text {. }
$$

In [8], Sivley introduced an LMS technique to produce an unwrapped phase estimate $\widehat{\varphi}_{L M S_{k}}(\mathbf{x})$ for the AM-FM model having a second derivative as close as possible to the second derivative of the principal phase in a least-squares sense. However, $\widehat{\varphi}_{L M S_{k}}(\mathbf{x})$ is not itself congruent with the principal phase. He then computed a congruent unwrapped phase from $\widehat{\varphi}_{L M S_{k}}(\mathbf{x})$ through the use of a scaling factor (referred to as phase embedding in [8]). Upon multiplying $\widehat{\varphi}_{L M S_{k}}(\mathbf{x})$ by a sufficiently large scale factor and then selecting the closest congruent phase values, a congruent unwrapped phase is created that retains the structure of the LMS estimate in a scaled form.

When differentiated, this unwrapped phase provides a meaningful FM field that can be integrated to perfectly recover the (scaled) phase, provided that phase initial conditions are saved to disambiguate the integration constant. In practice, one generally needs to save multiple phase initial conditions on a grid so that the phase reconstruction can be periodically restarted to improve noise immunity and robustness. This approach works well as long as FM filtering is not applied. However, if the FM functions have been modified then the phase initial conditions cannot be extracted directly from the the original image except in some restricted special cases (e.g., RST image rotation, scaling, translation) $[10,11]$.

Successful reconstruction after more general FM processing was demonstrated in [10], but revealed a fidelity loss that can be substantial due to the phase scaling used in the unwrapping algorithm of [8]. At the heart of this problem is the fact that phase scaling renders the phase reconstruction extremely sensitive to estimation errors in the initial phase values. With a scale factor of 300 (typical), small deviations in the estimates are greatly amplified and then contribute to the overall image reconstruction by a translation of the reconstructed phase surface that is projected through the trigonometric functions in (1). While this is of little consequence for reconstructing an unprocessed image where the initial phase values are accurately known, it can seriously degrade the reconstruction fidelity in cases where the initial phase values are unknown and must be estimated subsequent to modification of the FM functions by filtering.

\section{FREQUENCY GUIDED PHASE UNWRAPPING}

Phase unwrapping techniques may be generally classified as either path-following algorithms or minimum-norm algorithms $[8,17]$. The new phase unwrapping method introduced here uses elements of both approaches. It employs the LMS unwrapped estimates described in [8], but uses a region growing technique similar to those found in $[14,15,17,18]$ to obtain a congruent unwrapped phase that is also unscaled, has minimal local variation, and closely agrees with the smooth (but incongruent) LMS phase estimate. As in [8], the LMS unwrapped phase estimates are computed on the pixel grid using a DCT-based technique. However, unlike [8] where phase scaling is applied to impose congruency, here the LMS solution is directly interpolated with cubic tensor product splines and differentiated analytically to obtain an initial estimate of the FM field. This initial estimate is in good agreement with visual perception but cannot provide PR because the LMS phase estimates are incongruent with the principal phase values.

The initial FM estimate is used to guide a queue-based region growing algorithm in order to obtain an improved unwrapped phase solution $\widehat{\varphi}_{k}$ that is congruent with the principal phase but has a gradient that is close to the initial FM estimate in the sense that it is also in good agreement with visual perception of the image. The region growing algorithm seeks to unwrap phase values smoothly starting from dominant/salient seed points characterized by high amplitude. For each component $t_{k}$ in (1), the AM function $a_{k}$ is thresholded at $20 \%$ of peak. Connected components labeling is performed on the set of pixels where the AM exceeds this threshold. For each resulting connected component, the pixel with maximum AM is placed in a queue where it will seed a region in the final unwrapped phase solution. For each of these seed pixels, the final phase solution is computed by (4) with

$$
b=\underset{b \in \mathbb{Z}}{\arg \min }\left|\left(\varphi_{k}(\mathbf{x})+2 \pi b\right)-\widehat{\varphi}_{L M S_{k}}(\mathbf{x})\right|,
$$

which is the congruent phase value that is closest in absolute value to the LMS unwrapped phase. The seed pixel is then removed from the head of the queue and its 4-neighbors are added to the tail of the queue. When all seed pixels have been processed in this way, the queue is initialized.

Phase unwrapping is then performed for all remaining pixels in $t_{k}$ using the new region growing algorithm given in Table 1 . In each iteration of the main Repeat loop, an improved unwrapped phase

Table 1. Queue-based phase region growing algorithm

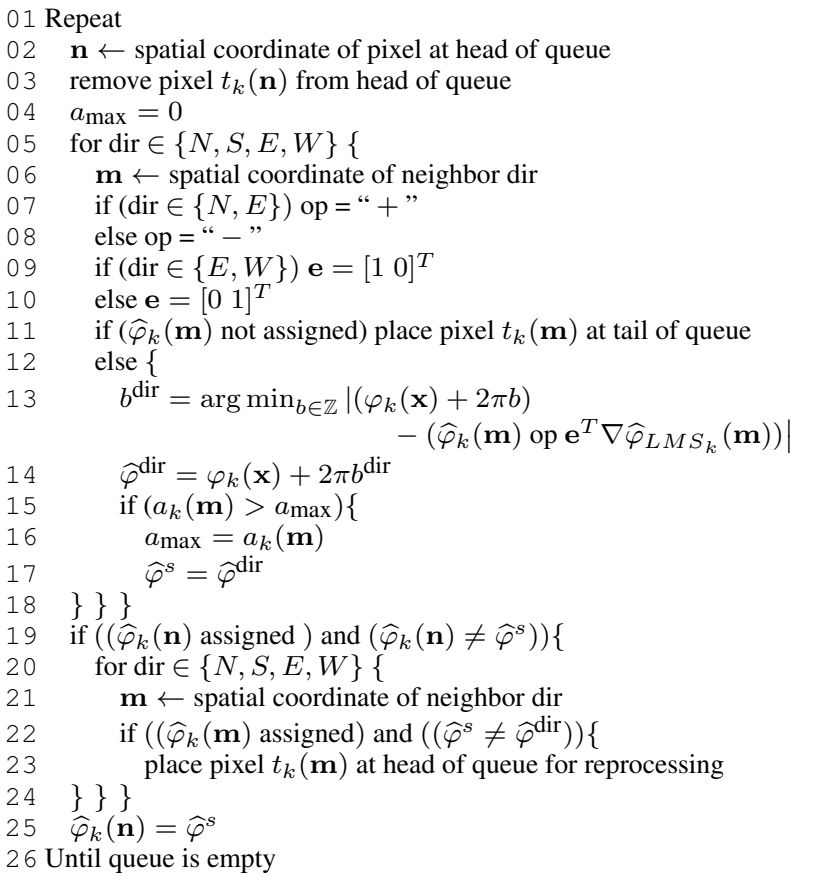


value is assigned to the pixel $t_{k}(\mathbf{n})$ at the head of the queue. This is accomplished by looping over the $N, S, E$, and $W$ neighbor pixels. For each neighbor $t_{k}(\mathbf{m})$ where an unwrapped phase has already been assigned, lines 13-14 select a candidate congruent phase value $\widehat{\varphi}^{\text {dir }}$ with minimum absolute difference from the sum (for a $N$ or $E$ neighbor) or difference (for a $S$ or $W$ neighbor) of the appropriate (horizontal or vertical) component of the LMS frequency estimate at $\mathbf{m}$ and the unwrapped phase at $\mathbf{m}$. These candidates will all be congruent, differing by integer multiples of $2 \pi$ as a consequence of lying on different branches of arctan in (3). Lines 17 and 25 assign the unwrapped phase at $\mathbf{n}$ by selecting the candidate with largest AM, which tends to suppress branch cuts from occurring in neighborhoods where the amplitude is large. Any 4-neighbors of pixel $t_{k}(\mathbf{n})$ where an unwrapped phase has not been assigned are added to the tail of the queue for subsequent processing (line 11).

The conditional loop in lines 19-24 identifies 4-neighbors of pixel $t_{k}(\mathbf{n})$ where a suboptimal unwrapped phase was previously assigned by using the wrong neighbor $\widehat{\varphi}_{k}(\mathbf{m})$ in line 13 . This occurs because the unwrapped phase at the best neighbor was not yet available due to the pixel processing order. To improve local smoothness and redirect branch cuts towards local amplitude minima, we return such 4-neighbors to the head of the queue for immediate reprocessing (line 23). The test in line 19 avoids the possibility of an infinite recursion by ensuring that the neighbors of any such requeued pixel will not themselves be returned to the queue unless a previously assigned phase value is actually changed.

When the algorithm terminates, the unwrapped phase is congruent with the principal values and agrees closely with the LMS estimate while avoiding the use of phase embedding (scaling) as in [8]. Because of nonconservative residues in the phase [18], undesirable branch cuts still arise as shown in Fig. 1(b). However, this new algorithm tends to place them along minimum amplitude paths where their contribution to the overall image reconstruction is small. For each image component $t_{k}$ in (1), the FM function $\nabla \varphi_{k}(\mathbf{x})$ is then obtained by fitting the unwrapped phase samples with cubic tensor product splines and differentiating analytically.

\section{ESTIMATION OF INITIAL PHASE VALUES}

Reconstruction from the AM-FM model is performed by reconstructing the phase of each component via spline-based integration of $\nabla \varphi_{k}[8,19]$ and then applying (1). Integration along any row or column requires two adjacent phase initial conditions. Most AMFM reconstruction techniques save initial phase values from the original image as side information for this purpose. However, if FM processing has been applied this approach will not work in general because the initial phase values of the processed image are unknown. This issue was mitigated in [11] by encoding a DC component into $a_{k}$ from which the initial phase values could be reconstructed. Here, we approach the problem by retaining only a single phase initial condition $\widehat{\varphi}_{k}\left(\mathbf{n}_{1}\right)$, where $\mathbf{n}_{1}=\left(r_{1}, c_{1}\right)$ is the pixel coordinate of the global max of $a_{k}(\mathbf{n})$. We then estimate the remaining required phase initial conditions directly from the potentially modified FM field as follows.

For $\mathbf{n}_{2}=\left(r_{1}, c_{1}-1\right), \mathbf{n}_{3}=\left(r_{1}-1, c_{1}-1\right)$, and $\mathbf{n}_{4}=$ $\left(r_{1}-1, c_{1}\right)$, we add appropriate frequency estimates from $\nabla \varphi_{k}(\mathbf{n})$ to $\widehat{\varphi}_{k}\left(\mathbf{n}_{1}\right)$. With these four initial phase values, spline integration can be applied to reconstruct $\widehat{\varphi}_{k}$ on the entire region of $t_{k}$ lying above and to the left of $\mathbf{n}_{1}$ by first integrating up columns $c_{1}-1$ and $c_{1}$ and then integrating from right to left along rows from $r_{1}$ to the top of the image. To reconstruct the phase on the region of $t_{k}$ lying below and to the left of $\mathbf{n}_{1}$, we integrate down columns
Table 2. Mean squared reconstruction error for chirp, lines, and lena images using three different AM-FM reconstruction methods.

\begin{tabular}{|l|c||c|c|c|}
\hline \multirow{2}{*}{ Algorithm } & \multicolumn{1}{|c||}{$\begin{array}{c}\text { Initial } \\
\text { Phase Vals }\end{array}$} & \multicolumn{3}{c|}{ MS Reconstruction Error } \\
\cline { 3 - 5 } & shirp & lines & lena \\
\hline \hline Phase Scaling [8] & stored & $10^{-8}$ & $10^{-8}$ & $10^{-8}$ \\
\hline Proposed & stored & $10^{-11}$ & $10^{-12}$ & $10^{-12}$ \\
\hline Proposed & estimated & $10^{-6}$ & $10^{-6}$ & $10^{-5}$ \\
\hline
\end{tabular}

$c_{1}-1$ and $c_{1}$ and then across rows from $r_{1}+1$ to the bottom of the image. Analogous procedures are used to reconstruct $\widehat{\varphi}_{k}$ on the regions lying above and to the right of $\mathbf{n}_{1}$ and below and to the right of $\mathbf{n}_{1}$.

\section{EXAMPLES \& DISCUSSION}

The motivation for our proposed new phase unwrapping and initial phase value estimation techniques was to develop a suitable means for AM-FM reconstruction of a processed image after modification of the FM functions by modulation domain filtering. However, in this section we demonstrate the proposed techniques on the three unfiltered images given in Fig. 1(d)-(f), since this provides known ground truth thereby enabling quantitative evaluation of the reconstruction errors. For each image, we computed an AM-FM model with $K=40$ in (1). We reconstructed using the phase scaling method given in [8], the proposed method with multiple initial phase values stored, and the proposed method with only a single initial phase value stored and the rest computed by the technique given in Section 4. Mean squared reconstruction errors for each case are given in Table 2 for floating point pixels in the range [-1,1] (the pixels were scaled for modeling against (1)). For all nine cases given in Table 2, the reconstructed images were visually indistinguishable from the originals.

Component $t_{20}$ of the chirp image is given in Fig. 1(a). The unwrapped phase computed by the proposed method is given in Fig. 1(b), where branch cuts are visible in the low-amplitude, lowsaliency regions. The FM field $\nabla \varphi_{20}(\mathbf{x})$ obtained by differentiating the unwrapped phase is given in Fig. 1(c). Fig 1(d) shows the reconstruction obtained by adding all 40 components reconstructed by the technique of Section 4. Visually, it is indistinguishable from the original. Similar high fidelity reconstructions for lines and lena are given in Fig. 1(e) and (f). The main significance of these results is twofold. Line two of Table 2 demonstrates that the proposed phase unwrapping technique substantially improves the quality of the computed FM field. Line three shows that the proposed initial phase value estimation technique is capable of delivering high fidelity image reconstructions without the need for saving multiple phase initial conditions, which is important for reconstructing from filtered AM and FM functions where the true initial phase values are unknown.

\section{REFERENCES}

[1] T. Tangsukson and J. P. Havlicek, "AM-FM image segmentationn," in Proc. IEEE Int'l. Conf. Image Proc., Vancouver, BC, Canada, Sep. 10-13, 2000, vol. II, pp. 104-107.

[2] J. P. Havlicek, J. Tang, S. T. Acton, R. Antonucci, and F. N. Ouandji, "Modulation domain texture retrieval for CBIR in 

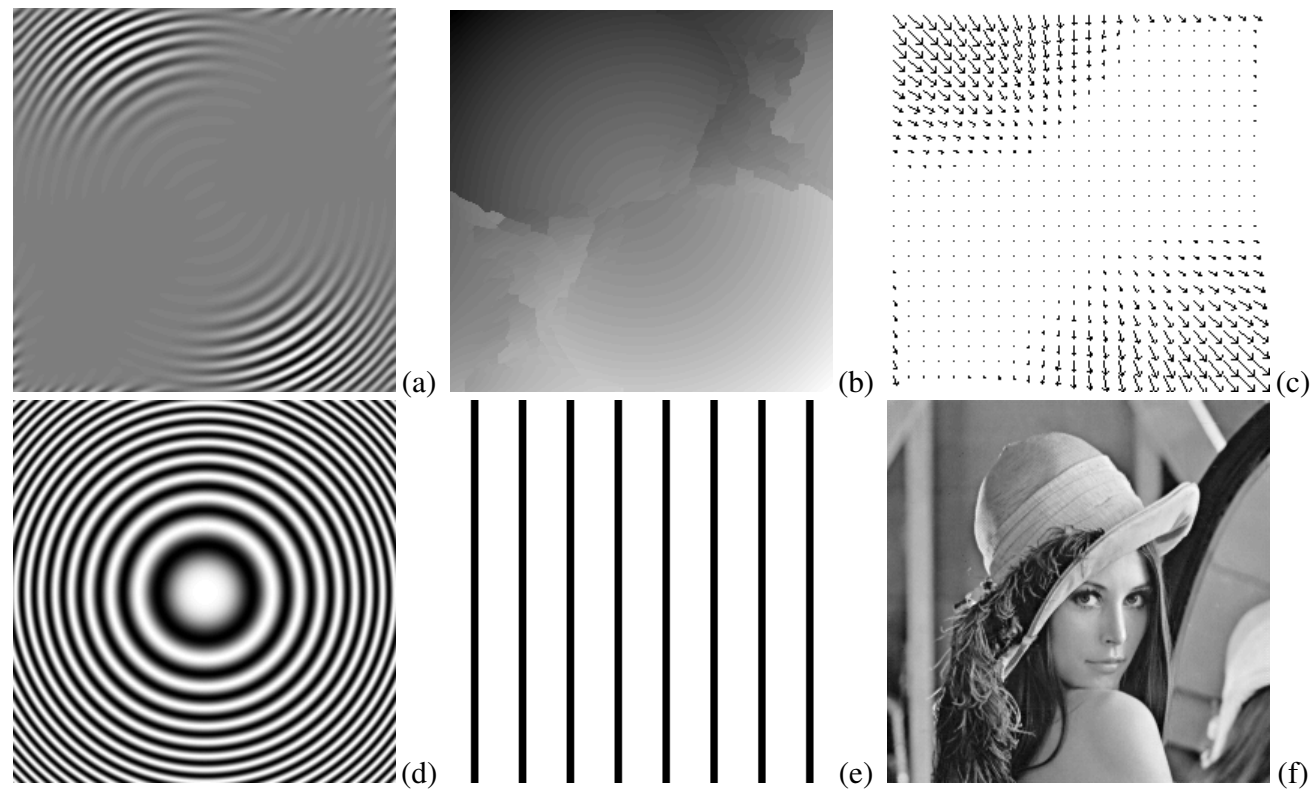

Fig. 1. Phase unwrapping and reconstruction examples. (a) Component $t_{20}$ of chirp image. (b) Unwrapped phase from (a). (c) FM function $\nabla \varphi_{20}$ obtained by differentiating (b). (d) Reconstructed chirp image. (e) Reconstructed lines image. (f) Reconstructed lena image.

digital libraries," in Proc. 35th IEEE Asilomar Conf. Signals, Syst., Comput., Nov. 9-12, 2003, pp. 1580-1584.

[3] R. S. Prakash and R. Aravind, "Modulation-domain particle filter for template tracking," in Proc. Int'l. Conf. Pattern Recog., Tampa, FL, Dec. 8-11, 2008, pp. 1-4.

[4] M. Shook, J. Junger, N. Mould, and J.P. Havlicek, "Quantifying infrared target signature evolution using AM-FM features," in Proc. IEEE Southwest Symp. Image Anal., Interp., 2010, pp. 189-192.

[5] C.C. Loizou, V. Murray, M.S. Pattichis, M. Pantziaris, and C.S. Pattichis, "Multiscale amplitude-modulation frequencymodulation (AM-FM) texture analysis of ultrasound images of the intima and media layers of the carotid artery," IEEE Trans. Inf. Technol. Biomed., vol. 15, no. 2, pp. 178-188, Mar. 2011.

[6] C.C. Loizou, V. Murray, M.S. Pattichis, I. Seimenis, M. Pantziaris, and C.S. Pattichis, "Multiscale amplitudemodulation frequency-modulation (AM-FM) texture analysis of multiple sclerosis in brain MRI images," IEEE Trans. Inf. Technol. Biomed., vol. 15, no. 1, pp. 119-129, Jan. 2011.

[7] J. P. Havlicek, D. S. Harding, and A. C. Bovik, "Multidimensional quasi-eigenfunction approximations and multicomponent AM-FM models," IEEE Trans. Image Proc., vol. 9, no. 2, pp. 227-242, Feb. 2000.

[8] R. A. Sivley and J. P. Havlicek, "Multidimensional phase unwrapping for consistent APF estimation," in Proc. IEEE Int'l. Conf. Image Proc., Genoa, Italy, Sep. 11-14, 2005, vol. II, pp. $458-461$.

[9] C. T. Nguyen and J. P. Havlicek, "AM-FM image filters," in Proc. IEEE Int'l. Conf. Image Proc., San Diego, CA, Oct. 1215, 2008, pp. 789-792.

[10] C. T. Nguyen, P. A. Campbell, and J. P. Havlicek, "FM filters for modulation domain image processing," in Proc. IEEE Int'l. Conf. Image Proc., Cairo, Egypt, 2009, pp. 3973-3976.
[11] C. T. Nguyen, J. D. Williams, J. P. Havlicek, and M. Ozaydin, "FM processing with generalized amplitude \& phase: Application to modulation domain geometric image transformations," in Proc. IEEE Int'l. Conf. Image Proc., 2011, pp. 81-84.

[12] C.T. Nguyen and J.P. Havlicek, "AM-FM models, partial Hilbert transform, and the monogenic signal," in Proc. IEEE Int'l. Conf. Image Proc., Orlando, FL, Sep. 30-Oct. 3, 2012, pp. 2337-2340.

[13] E. P. Simoncelli and W. T. Freeman, "The steerable pyramid: a flexible architecture for multi-scale derivative computation," in Proc. IEEE Int'l. Conf. Image Proc., Washington, DC., Oct. 23-26, 1995, pp. 444-447.

[14] G. Fornaro and E. Sansosti, "A two-dimensional region growing least squares phase unwrapping algorithm for interferometric SAR processing," IEEE Trans. Geoscience and Remote Sensing, vol. 37, no. 5, pp. 2215-2226, 1999.

[15] Wei Xu and I. Cumming, "A region-growing algorithm for InSAR phase unwrapping," IEEE Trans. Geoscience and Remote Sensing, vol. 37, no. 1, pp. 124-134, 1999.

[16] S. Moon-Ho Song, S. Napel, N.J. Pelc, and G.H. Glover, "Phase unwrapping of MR phase images using Poisson equation," IEEE Trans. Image Proc., vol. 4, no. 5, pp. 667-676, 1995.

[17] Heping Zhong, Jinsong Tang, Sen Zhang, and Ming Chen, "An improved quality-guided phase-unwrapping algorithm based on priority queue," Geoscience and Remote Sensing Letters, IEEE, vol. 8, no. 2, pp. 364-368, 2011.

[18] T. Flynn, "Consistent 2-D phase unwrapping guided by a quality map," in Proc. Int'l. Geoscience and Remote Sensing Symp., 1996, vol. 4, pp. 2057-2059 vol.4.

[19] M. Unser, A. Aldroubi, and M. Eden, "B-spline signal processing: Part I-theory," IEEE Trans. Signal Proc., vol. 41, no. 2, pp. 821-833, Feb. 1993. 